\title{
Evolution of Gas in Galaxies
}

\section{Lister Staveley-Smith ${ }^{* \dagger}$}

School of Physics, University of Western Australia, Crawley, WA 6009, Australia

E-mail: Lister.Staveley-Smith@uwa.edu.au

The SKA will be a unique instrument with which to study the evolution of the gas content of galaxies. A proposed deep ( $\sim 8 \mathrm{Msec}$ ) 'pencil-beam' survey is simulated using recently updated specifications for SKA sensitivity and survey speed. Almost $10^{7}$ galaxies could be detected in the redshifted $21 \mathrm{~cm}$ line, most at redshifts in excess of two. This will enable confident statements to be made about the evolution of the cosmic HI density and the HI mass function to $z=3$, corresponding to a lookback time of $11 \mathrm{Gyr}$. However, galaxies or groups of galaxies with masses the same as the most HI-massive galaxies at $z=0$ will be detectable at redshifts of 6 , if they exist. The ideal instrument for studying HI evolution would have an instantaneous sensitivity at least a factor of two higher than current specifications in the critical frequency range $200-500 \mathrm{MHz}$, or $A / T>2 \times 10^{4} \mathrm{~m}^{2} \mathrm{~K}^{-1}$. The capabilities of the SKA will be highly complementary to ALMA which will be able to study the evolution of the molecular gas component over the same redshift range.

From planets to dark energy: the modern radio universe October 1-5 2007

University of Manchester, Manchester, UK

\footnotetext{
* Speaker.

${ }^{\dagger}$ West Australian Premier's Fellow
} 


\section{Introduction}

Understanding the evolution of galaxies is one of the goals of modern cosmology and one of the five key science goals of the SKA [1]. A key ingredient in galaxy evolution is the gas supplied through various accretion, merger and feedback processes that occur during the assembly of galaxies. This gas, which is mainly hydrogen, passes through a neutral atomic phase and later condenses into massive molecular clouds and stars. During the atomic phase, it can readily be traced with the $21 \mathrm{~cm}$ hyperfine spin-flip transition and the Gunn-Peterson effect.

At high redshifts, $z>1.6$, neutral hydrogen is currently traced by ground-based observations of Gunn-Peterson absorption lines against bright background QSOs. Such observations demonstrate that the bulk of the neutral hydrogen is in the Damped Ly- $\alpha$ systems with column densities exceeding $2 \times 10^{20} \mathrm{~cm}^{-2}$. However, interpretation of these observations are hampered by insufficient lines of sight and serious uncertainties associated with dust obscuration, gravitational lensing and intrinsic source size. These uncertainties lead to contradictory results from measurements asso-

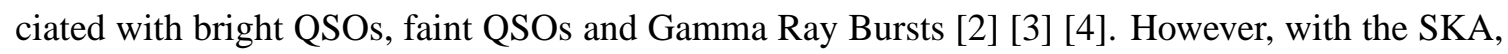
galaxies will be detected at similar redshifts in $21 \mathrm{~cm}$ line emission, which will lead to a clearer understanding of the distribution of gas in the Universe, and the manner in which the gas content of galaxies evolves with time.

In order to measure the gas content of galaxies at the highest redshift, a deep pencil beam survey with the SKA is proposed. Currently proposed SKA specifications [5] are adopted, and used to generate artificial galaxy catalogues which are used to simulate the accuracy with which one simple parameter can be recovered, namely the cosmic HI density - the comoving volume density of neutral hydrogen.

\section{An SKA Deep Field}

An early goal in the design and development of the SKA has been the requirement to detect and image galaxies beyond a redshift of unity in the redshifted $21 \mathrm{~cm}$ line of neutral hydrogen and in the radio continuum [6]. However, the astronomy community has since developed an impressive list of complementary science goals for the SKA, including the study of the early intergalactic medium, dark energy and cosmology, pulsars and tests of gravitational theory, planet formation and cosmic magnetism [1]. The resultant range of requirements (e.g. frequencies, baselines, field-of-view) has inevitably added to the cost of the SKA and created complexities in developing a design. A recent SKA project study [5] has recommended the adoption of a preliminary set of specifications for the SKA and its various phases. For the purpose of the discussion of a putative SKA deep field, these specifications are adopted in this paper. Where multiple specifications exist due to technology uncertainty, the specifications relating to the simplest technology has been chosen. The SKA specifications, although realistic, are nevertheless still indicative and are likely to change as the SKA design evolves. For reference, a summary of the adopted values, in the frequency range of interest, is listed in Table 1.

A critical requirement for the study of galaxy evolution is the ability to detect galaxies at the highest redshift. Since the bandwidth is defined by the Doppler width of the galaxies, this ability is only dependent on the instantaneous sensitivity $A / T$, and the integration time $\tau$. Unlike for many 


\begin{tabular}{lccccc}
\hline $\begin{array}{l}\text { Frequency } \\
\mathrm{MHz}\end{array}$ & $z$ & $\begin{array}{c}A / T^{1} \\
\mathrm{~m}^{2} \mathrm{~K}^{-1}\end{array}$ & $\begin{array}{c}\Omega \\
\mathrm{deg}^{2}\end{array}$ & $\begin{array}{c}\tau \\
\mathrm{s}\end{array}$ & $N$ \\
\hline $70-200$ & $6.1-19$ & 3000 & 200 & $8 \times 10^{6}$ & 0 \\
$200-500$ & $1.8-6.1$ & 7500 & 200 & $8 \times 10^{6}$ & $6.6 \times 10^{6}$ \\
$500-1000^{2}$ & $0.42-1.8$ & 9000 & 2.0 & $8 \times 10^{6}$ & $4.4 \times 10^{5}$ \\
$1000-1420^{2}$ & $0-0.42$ & 9000 & 0.4 & $8 \times 10^{6}$ & $2.1 \times 10^{4}$ \\
\hline
\end{tabular}

${ }^{1}$ Assumes that $75 \%$ of the SKA aperture will reside on baseline lengths short enough not to significantly resolve distant galaxies.

${ }^{2}$ Assumes wideband feed technology which gives a slightly better sensitivity $(A / T)$, but a dramatically worse field of view.

Table 1: The adopted set of SKA specifications used in the simulations, and the number of galaxies detectable in the simulations, in a deep-field HI survey. $z$ is the redshift range; $A / T$ is the telescope gain; $\Omega$ is the field of view; $\tau$ is the integration time; $N$ is the number of galaxy detections expected. The specifications follow the current SKA project office recommendations [5], but are subject to modification. There are a number of other assumptions made in simulating the numbers of galaxies detected in this survey, the most important ones being: that the comoving HI mass function and velocity function is described by HIPASS [7]; that the galaxy detection threshold is $5-\sigma$, and that the minimum resolution used for the detection algorithm is $0.1 \mathrm{MHz}$.

other science goals, 'survey' or 'mapping' speed is much less important as long as the field-of-view is able to deliver suitably large statistical samples. High mapping speed, on its own, is insufficient to ensure that the highest redshift galaxies can be detected.

For this paper, the sole question of the evolution of the cosmic HI density is addressed. This only requires the detection, not imaging, of galaxies. Therefore, somewhere between $50 \%$ (baselines less than $5 \mathrm{~km}$ ) and $75 \%$ (baselines less than $180 \mathrm{~km}$ ) of the full SKA collecting area is available, before galaxies become resolved and more difficult to detect. Here, it is assumed that $75 \%$ is available, corresponding to a sensitivity of $A / T=7500 \mathrm{~m}^{2} \mathrm{~K}^{-1}$ in the critical redshift range 1.8 - 6.1. Values for other frequencies are listed in Table 1. Low redshift galaxies remain heavily resolved. Nevertheless, at the higher redshifts of interest in this paper, where the great majority of the galaxies will be detected, the assumption remains reasonable. Available integration time is more difficult to judge but, given the wide range of science possibilities opened up with a deep field observation, it seems likely that a substantial integration time may be feasible. It is assumed that 8 Msec, which corresponds to $180 \times 12 \mathrm{hrs,} \mathrm{will} \mathrm{be} \mathrm{available} \mathrm{over} \mathrm{the} \mathrm{first} \mathrm{few} \mathrm{years} \mathrm{of} \mathrm{operation} \mathrm{of}$ the SKA.

\section{Results}

A simulated catalogue was created using the above telescope specifications and a non-evolving HIPASS HI mass function [7]. The redshift distribution is shown in Figure 1. The small field-ofview at frequencies from 500 to $1420 \mathrm{MHz}$ results in a relatively small number of objects detected at low redshifts. For example, only 21,000 galaxies are detected at redshifts beneath 0.42 , which is a factor of 10 less than expected from the proposed ASKAP deep survey ([\$] and Figure 1), despite the latter being conducted with a telescope of less than $1 \%$ of the SKA collecting area. However, a shallow SKA survey over a large area of sky is a better way of detecting larger numbers of lowredshift galaxies. At higher redshifts, much larger numbers of galaxies are detected, mainly due to 


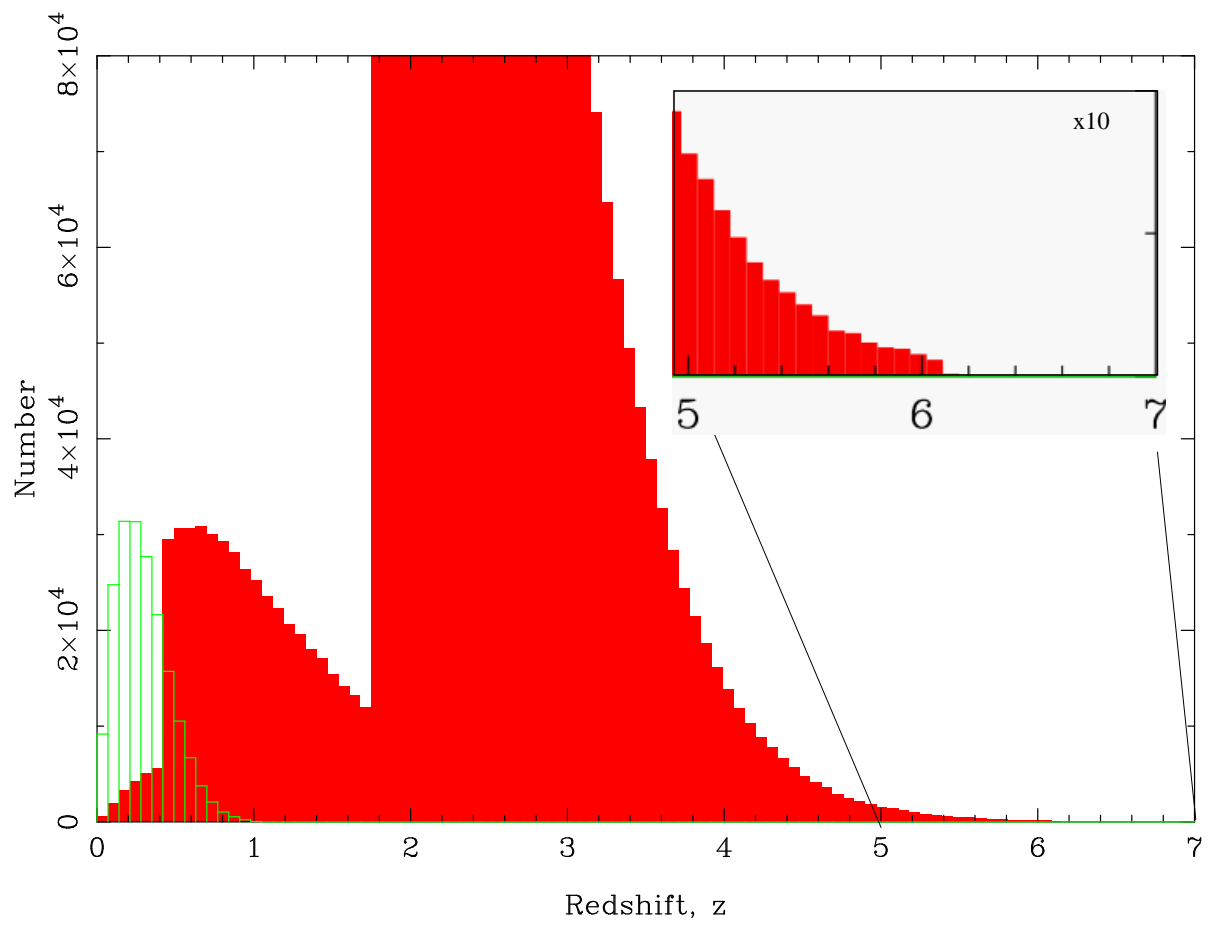

Figure 1: Solid (red) histogram: the number of galaxies expected to be detected in $\mathrm{HI}$ in different redshift bins from 0 to 7 in a deep SKA 'pencil beam' survey of integration time $8 \mathrm{Msec}$; open (green) histogram: the number of galaxies expected below $z=1$ in the proposed ASKAP deep survey (Johnston et al. 2007). Each redshift bin is of width $\Delta z=0.08$ and the total numbers of galaxies in different redshift intervals is listed in the previous table. The histogram is truncated due to the large numbers of galaxies near $z=2$.

the much larger cosmic volume surveyed. $M^{*}$ galaxies are seen to redshifts of about 3.4, beyond which the number of detected galaxies drops off dramatically.

For each redshift interval, the HI density integral $\int M_{H} \phi\left(M_{H}\right) d M_{H}$ is calculated for the detected galaxies and shown in Figure 2 . The 'detected' density remains within a factor of a few of the input density at redshift up to $\sim 2$, but drops to $10 \%$ at a redshift of $\sim 3.5$ as the bulk of the mass-bearing galaxies are too faint to detect. Nevertheless, using an assumption that the shape of the mass function is constant, it is possible to recover the input mass density even at redshift 5 (Figure 2) before shot noise errors become large. In practice, the shape of the mass function will change with redshift in an unknown manner, so it will be hard to reliably recover the cosmic HI mass density much beyond a redshift of 3 from emission measurements alone.

\section{Discussion}

This simulation has demonstrated the large numbers of high-redshift galaxies that can be detected by the SKA in a significant, but feasible, HI survey of galaxies, and has demonstrated the high accuracy with which the cosmic HI density can be measured. Whilst galaxy numbers are low at redshifts below 1.8, this is largely due to the small field of view available to single pixel technology. Adoption of widefield detection technology has the potential to greatly increase num- 


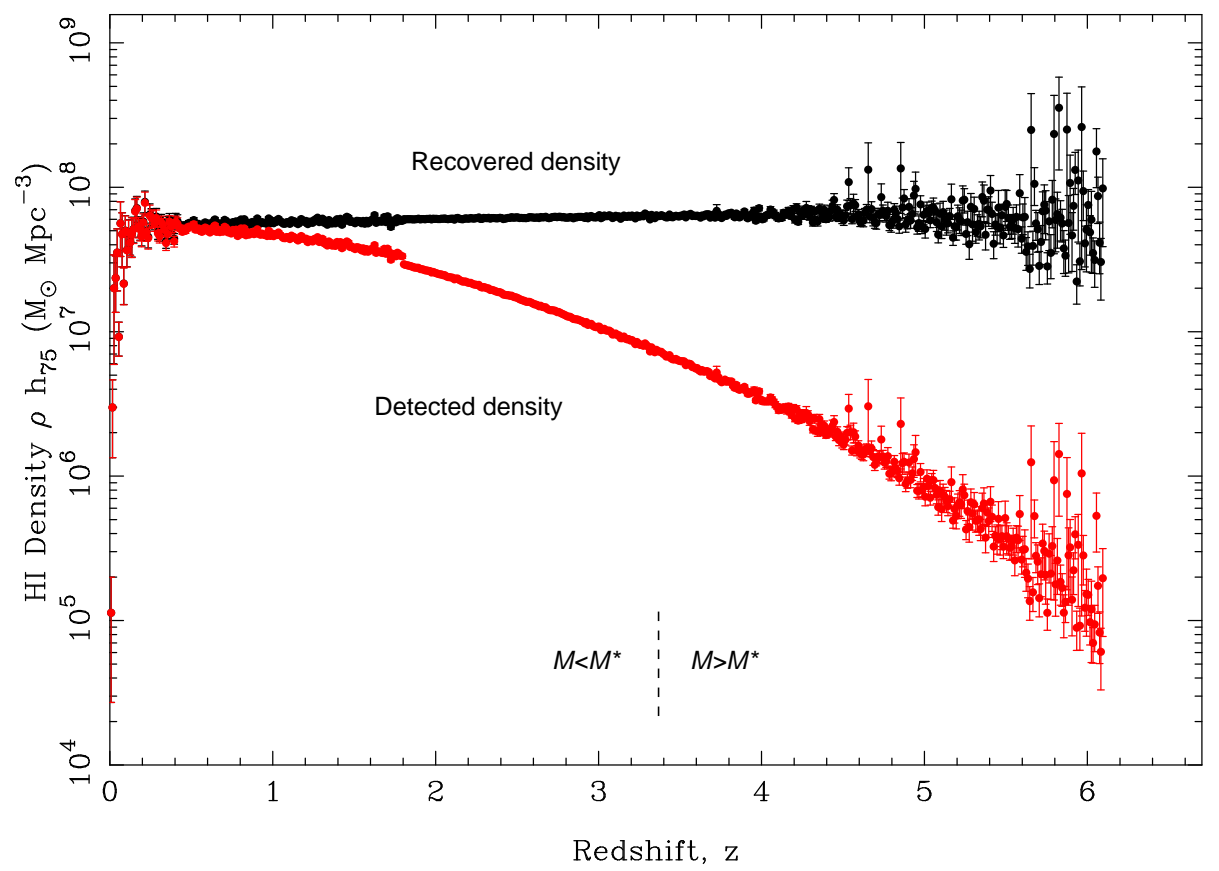

Figure 2: A simulation which shows how well the cosmic HI density can be recovered at various redshifts using the simulated galaxy catalogue of the previous figure. The upper (black) points show the recovered density, after correction for galaxies below the detection threshold; the lower (red) points show the measured density of directly detected galaxies only.

bers. Although the subsequent reduction of errors associated with shot noise and cosmic variance is important for many science goals, greater gains in the field of galaxy evolution study are likely to be made with better instantaneous sensitivity at lower frequencies. This will increase the ability to detect changes in the $\mathrm{HI}$ mass function and reduce the density extrapolation required to account for low-mass galaxies at redshifts approaching 3. An appropriate goal of $A / T>2 \times 10^{4} \mathrm{~m}^{2} \mathrm{~K}^{-1}$ is therefore suggested for frequencies below $500 \mathrm{MHz}$.

\section{References}

[1] Carilli C.L., Rawlings S. 2004, NewAR, 48, 979

[2] Prochaska J.X., Herbert-Fort, S., Wolfe A.M. 2005, ApJ, 635, 123

[3] Prochter G.E. et al. 2006, ApJ, 648, 93

[4] Porciani C., Viel M., Lilly S.J. 2007, ApJ, 659, 218

[5] Schilizzi R.T. et al. 2007, 'Preliminary Specifications for the Square Kilometre Array', version 2.7.1 (4 December 2007), SKA Project Office

[6] van der Hulst J.M., Sadler E.M., Jackson C.A., Hunt L.K., Verheijen M., van Gorkom J.H. 2004, NewAR, 48, 1221

[7] Zwaan M.A., Meyer M.J., Staveley-Smith L., Webster R.L. 2005, MNRAS, 359, 30

[8] Johnston S. et al. 2007, PASA, 24, 174 